\title{
A dichotomy theorem for mono-unary algebras
}

\author{
by
}

Su G a o (Pasadena, CA)

\begin{abstract}
We study the isomorphism relation of invariant Borel classes of countable mono-unary algebras and prove a strong dichotomy theorem.
\end{abstract}

1. Introduction. In this paper we consider countable mono-unary algebras. A mono-unary algebra used to be defined as a pair $\langle A, f\rangle$ where $A$ is a set and $f$ is a unary function from $A$ into $A$. But here we shall consider a slightly more general kind of structures, namely for which $f$ is a partial unary function on $A$. In model theoretic terms, let us fix a language $L=\{R\}$, where $R$ is a binary relation symbol, and consider countable models of language $L$ with the property

$$
\forall x \forall y \forall z(R(x, y) \wedge R(x, z) \Rightarrow y=z) .
$$

Then the binary relation $R$ can be viewed as a partial unary function on the model, and we call such models mono-unary algebras as well.

We denote by $\mathbb{U}$ the space of all countable mono-unary algebras with domain $\omega$, where $\omega$ is the set of all finite ordinals. Each model in $\mathbb{U}$ is naturally coded by an element of $2^{\omega \times \omega}$, thus by a real in the Cantor space. It follows that the Borel structure of the Cantor space gives rise to a Borel structure on $\mathbb{U}$. Moreover, this Borel structure can be induced by a topology that is Polish, i.e., complete metric and separable (see [Sa]). The isomorphism relation among all countable mono-unary algebras is now regarded as an equivalence relation on the Polish space $\mathbb{U}$. It is easy to check that this equivalence relation is $\boldsymbol{\Sigma}_{1}^{1}$.

Let $L_{\omega_{1} \omega}$ be the infinitary language formed under first-order formation rules augmented by countable length conjunctions and disjunctions. For a

2000 Mathematics Subject Classification: Primary 03E15, 08A60; Secondary 03C15, $03 \mathrm{C} 70$.

Key words and phrases: descriptive set theory, countable model theory, admissible set theory. 
sentence $\sigma$ in $L_{\omega_{1} \omega}$, the space of all models of $\sigma$ with domain $\omega$ is denoted by $\operatorname{Mod}(\sigma)$. This is a Borel subset of the Polish space $\mathbb{U}$; and, following [FS], we say that $\operatorname{Mod}(\sigma)$ is an invariant Borel class. The isomorphism relation on $\operatorname{Mod}(\sigma)$, denoted by $\cong_{\sigma}$, is then a $\boldsymbol{\Sigma}_{1}^{1}$ equivalence relation on the standard Borel space $\operatorname{Mod}(\sigma)$.

The purpose of this paper is to investigate the complexity of the isomorphism relation $\cong_{\sigma}$ for various $\sigma$. For this we use Borel reducibility to compare the complexities of two equivalence relations. Suppose $X$ and $Y$ are standard Borel spaces and $E$ and $F$ are equivalence relations on $X$ and $Y$, respectively. $E$ is said to be Borel reducible to $F$, denoted $E \leq_{B} F$, if there is a Borel function $\theta: X \rightarrow Y$ such that, for any $x, y \in X$,

$$
x E y \Leftrightarrow \theta(x) F \theta(y) .
$$

If $\mathbb{A}$ and $\mathbb{B}$ are two invariant Borel classes of countable models, then $\mathbb{A}$ is Borel reducible to $\mathbb{B}$, denoted $\mathbb{A} \leq_{B} \mathbb{B}$, if $\cong \backslash \mathbb{A} \leq_{B} \cong \mid \mathbb{B}$ as equivalence relations. The notion is closely related to classification problems. In general, if $\cong_{\sigma} \leq_{B} \cong_{\sigma^{\prime}}$, then countable models of $\sigma$ are considered no more difficult to classify than those of $\sigma^{\prime}$, since any classification of $\operatorname{Mod}\left(\sigma^{\prime}\right)$ gives rise to a classification of $\operatorname{Mod}(\sigma)$. An invariant Borel class of countable models is Borel complete if any other invariant Borel class is Borel reducible to it. As noted in [FS] the isomorphism relation on a Borel complete class must be $\boldsymbol{\Sigma}_{1}^{1}$-complete as a subset of the (two-dimensional) Cantor space.

The main theorem in this paper is the following.

THEOREM 1.1. Let $\sigma$ be an $L_{\omega_{1} \omega}$ sentence all of whose models are monounary algebras. Then either $\cong_{\sigma}$ is Borel or else $\operatorname{Mod}(\sigma)$ is Borel complete.

The proof of the main theorem will actually establish a stronger result for first-order theories of mono-unary algebras.

TheOREM 1.2. Let $T$ be a first-order theory of mono-unary algebras. Then the following statements are equivalent:

(i) There is a model $M$ of $T$ with $\operatorname{sr}(M)=\omega_{1}^{M}$.

(ii) For any ordinal $\alpha<\omega_{1}$, there is a model $M$ of $T$ with $\operatorname{sr}(M)=$ $\omega_{1}^{M}>\alpha$.

(iii) $\cong_{T}$ is non-Borel.

(iv) $\operatorname{Mod}(T)$ is Borel complete.

In the statements, $\operatorname{sr}(M)$ stands for the Scott rank of $M$, and $\omega_{1}^{M}$ stands for the first admissible ordinal relativized to the real coding $M$. The definitions and properties of these concepts can be found in [Ba], Chapter VII.

Our main theorem generalizes earlier work on the number of countable mono-unary algebras. 
Corollary 1.3 (Vaught Conjecture, Marcus-Miller-Steel). Let $\sigma$ be an $L_{\omega_{1} \omega}$ sentence all of whose models are mono-unary algebras. Then $\sigma$ has either countably many or perfectly many models up to isomorphism.

Corollary 1.4 (The Glimm-Effros dichotomy). Let $\sigma$ be an $L_{\omega_{1} \omega}$ sentence all of whose models are mono-unary algebras. Then exactly one of the following is true:

$(\mathrm{I}) \cong_{\sigma} \leq_{B} \mathrm{id}(\mathbb{R})$, where $\mathrm{id}(\mathbb{R})$ is the identity relation on $\mathbb{R}$.

(II) $E_{0} \leq_{B} \cong_{\sigma}$, where $E_{0}$ is the Vitali equivalence relation on $\mathbb{R}$ defined, for any $x, y \in \mathbb{R}$, by $x E_{0} y \Leftrightarrow x-y \in \mathbb{Q}$.

To present the proofs we will also work with a concept which is closely related to mono-unary algebras, defined below. A structure $\mathcal{T}=\left\langle T,<^{\mathcal{T}}\right\rangle$ is called a tree if $<^{\mathcal{T}}$ is a partial order on $T$ such that for any $t \in T,<^{\mathcal{T}}$ linearly orders the set $\left\{s \in T \mid s<^{\mathcal{T}} t\right\}$. A countable tree $\mathcal{T}$ is called simple if it has a least element and for any $t \in T$, the set $\left\{s \in T \mid s<^{\mathcal{T}} t\right\}$ is finite. Note that our general concept of trees is quite different from that in graph theory, where a tree is defined to be an acyclic graph. The major difference is that our trees contain all linear orderings, which is not the case for graph theoretic trees. The simple trees are closer to the graph theoretical concept. One can view a simple tree as just a graph theoretic tree with a distinguished element, namely the root. In fact simple trees were called countable rooted trees of height $\leq \omega$ in [FS]. If we consider the partial function of finding the parent for each node in a simple tree, simple trees can be regarded as countable mono-unary algebras. We denote the space of all simple trees by $\mathbb{T}_{\omega}$ (following the phrasing of $[\mathrm{FS}]$ ). Then $\mathbb{T}_{\omega}$ is an invariant Borel subset of $\mathbb{U}$.

The interest in these concepts arose from earlier results in model theory and descriptive set theory. On the model theory side, the first-order Vaught's conjecture for mono-unary algebras was first established independently by Miller (unpublished) and Marcus ([Ma]). Later Steel [St] proved the infinitary version of Vaught Conjecture for trees.

On the descriptive set theory side, Friedman and Stanley studied in [FS], among other things, the structure of $\cong \uparrow \mathbb{T}_{\omega}$ and showed that $\mathbb{T}_{\omega}$ is Borel complete. It follows that $\mathbb{U}$ is also Borel complete. They also defined an $\omega_{1}$-sequence $\left\{\mathbb{S}_{\alpha}\right\}_{\alpha \in \omega_{1}}$ of invariant Borel classes of simple trees. Recall the following definition of $\alpha$-completeness: an invariant Borel class $\mathbb{A}$ is $\alpha$ complete if $\mathbb{S}_{\alpha} \leq_{B} \mathbb{A}$. A basic question of the subject is whether an invariant Borel class is Borel complete if it is $\alpha$-complete for all $\alpha<\omega_{1}$. Our theorem answers the question for invariant Borel classes of mono-unary algebras.

COROLlary 1.5. Let $\mathbb{A}$ be an invariant Borel class of mono-unary algebras. If $\mathbb{A}$ is $\alpha$-complete for all $\alpha<\omega_{1}$, then $\mathbb{A}$ is Borel complete. 
For the history of Glimm-Effros dichotomy theorems for equivalence relations, see e.g. [HKL] and [BK]. The classical theorem of [HKL] states that all Borel equivalence relations satisfy the Glimm-Effros dichotomy.

The rest of this paper is organized as follows. In Sections 2 and 3 we develop the technicalities needed for the proof of the main theorem. In particular, we establish the dichotomy for simple trees. Then in Section 4 we prove the main theorem. In the last section we derive some corollaries and give some remarks about further questions.

The work presented here is a part of the doctoral dissertation the author submitted to UCLA in 1998. The research is partially supported by 1997-98 Alfred Sloan Dissertation Fellowship. The author thanks his thesis advisor Greg Hjorth for many helpful conversations and the referee for the comments on an earlier draft of this paper.

2. Scott ranks and automorphisms of simple trees. In this section we concentrate on simple trees and prove some lemmas on the Scott ranks and automorphisms. The aim is to prepare enough terminology and facts for the proof of the key lemma in the next section. However, some of the lemmas proved here might also be interesting in their own right. First let us introduce some ad hoc notation for simple trees.

Let $\mathcal{T}$ be a simple tree and $t$ be an element of $\mathcal{T}$. We let $\mathcal{T}_{t}$ denote the structure $\left(\left\{s \in \mathcal{T} \mid t \leq^{\mathcal{T}} s\right\},<^{\mathcal{T}}\right)$, that is, the subtree of $\mathcal{T}$ rooted by $t$. Then $\mathcal{T}_{t}$ is also a simple tree. We define the level of $t, l(t)$, to be the cardinality of the set $\left\{s \in \mathcal{T} \mid s<^{\mathcal{T}} t\right\}$. The parent of $t, p(t)$, is the immediate predecessor of $t$ in $\mathcal{T}$. An element $s \in \mathcal{T}$ is a child of $t$ if $p(s)=t$. For any $t_{1}, t_{2} \in \mathcal{T}$, the meet of $t_{1}$ and $t_{2}, m\left(t_{1}, t_{2}\right)$, is defined to be the largest element $s \in \mathcal{T}$ with $s \leq^{\mathcal{T}} t_{1}$ and $s \leq^{\mathcal{T}} t_{2}$. If $m\left(t_{1}, t_{2}\right)$ is neither $t_{1}$ nor $t_{2}$, then $t_{1}$ and $t_{2}$ are incomparable. For $n \geq 1$, we define an equivalence relation $\sim$ on all $n$-tuples of $\mathcal{T}$ as follows. For $\vec{s}=\left(s_{0}, \ldots, s_{n-1}\right)$ and $\vec{t}=\left(t_{0}, \ldots, t_{n-1}\right)$,

$\vec{s} \sim \vec{t} \Leftrightarrow$ there is an automorphism $\tau: \mathcal{T} \rightarrow \mathcal{T}$ such that $\tau(\vec{s})=\vec{t}$, i.e., $\tau\left(s_{i}\right)=t_{i}$ for all $i<n$.

The automorphism $\tau$ in the definition is called a witness for $\vec{s} \sim \vec{t}$.

Lemma 2.1. Let $\mathcal{T}$ be a simple tree and $\vec{s}, \vec{t}$ be $n$-tuples of elements of $\mathcal{T}$. Then $\vec{s} \sim \vec{t}$ if and only if both the following conditions hold:

(i) For any $i<n, s_{i} \sim t_{i}$.

(ii) For any $i, j<n, l\left(m\left(s_{i}, s_{j}\right)\right)=l\left(m\left(t_{i}, t_{j}\right)\right)$.

Proof. The "only if" direction is obvious. For the "if" direction, we proceed by induction on $n$. Choose $\tau$ witnessing $\left(s_{0}, \ldots, s_{n-2}\right) \sim\left(t_{0}, \ldots, t_{n-2}\right)$. Let $s \in \mathcal{T}$ be maximal $\leq s_{n-1}$ with $s \leq s_{i}$ for some $i<n-1$. Let $t$ be the corresponding element $\leq t_{n-1}$. If $s=s_{n-1}$, then $t=t_{n-1}$ and $\tau$ witnesses 
$\vec{s} \sim \vec{t}$. Assume $s<s_{n-1}$ and $t<t_{n-1}$, and let $s^{\prime} \leq s_{n-1}, t^{\prime} \leq t_{n-1}$ be children of $s, t$ respectively. Choose $\tau^{\prime}$ witnessing $s_{n-1} \sim t_{n-1}$. So $\tau^{\prime}\left(s^{\prime}\right)=t^{\prime}$. We define $\tau^{\prime \prime}$ witnessing $\vec{s} \sim \vec{t}$.

If $\tau\left(s^{\prime}\right)=t^{\prime}$, then let

$$
\tau^{\prime \prime}=\tau^{\prime}\left|\mathcal{T}_{s^{\prime}} \cup \tau\right|\left(\mathcal{T} \backslash \mathcal{T}_{s^{\prime}}\right) .
$$

Otherwise, let $t^{*}=\tau\left(s^{\prime}\right)$ and $s^{*}=\tau^{-1}\left(t^{\prime}\right)$. Then $\tau^{*}=\tau\left(\tau^{\prime}\right)^{-1} \tau$ witnesses $s^{*} \sim t^{*}$. In this case let

$$
\tau^{\prime \prime}=\tau^{\prime}\left|\mathcal{T}_{s^{\prime}} \cup \tau^{*}\right| \mathcal{T}_{s^{*}} \cup \tau \mid\left(\mathcal{T} \backslash\left(\mathcal{T}_{s^{\prime}} \cup \mathcal{T}_{s^{*}}\right)\right) .
$$

In both cases $\tau^{\prime \prime}$ witnesses $\vec{s} \sim \vec{t}$.

The following lemma relates the above study of automorphisms to the Scott ranks of simple trees. For a comprehensive treatise of Scott analysis see [Ba]. For a simple tree $\mathcal{T}$, we denote the Scott rank of $\mathcal{T}$ by $\operatorname{sr}(\mathcal{T})$. If $\alpha$ is an ordinal and $\vec{t} \in \mathcal{T}$, we denote the canonical Scott $\alpha$-type of $\vec{t}$ by $\varphi_{\alpha}^{\vec{t}, \mathcal{T}}$. The canonical Scott sentence is denoted by $\varphi^{\mathcal{T}}$. Sometimes the superscipt $\mathcal{T}$ is omitted if there is no confusion. We let $\omega_{1}^{\mathcal{T}}$ denote the ordinal height of the least admissible set $\mathbf{A}$ containing $\mathcal{T}$, that is, $\mathbf{A}=L_{\omega_{1}^{\mathcal{T}}}[\mathcal{T}]$. By a well known result of Nadel $\operatorname{sr}(\mathcal{T}) \leq \omega_{1}^{\mathcal{T}}$. The countable fragment of $L_{\omega_{1} \omega}$ within A is then denoted by $L_{\mathbf{A}}$.

Lemma 2.2. Let $\mathcal{T}$ be a simple tree and $\mathbf{A}$ be the least admissible set containing $\mathcal{T}$. The following are equivalent:

(i) $\operatorname{sr}(\mathcal{T})<\omega_{1}^{\mathcal{T}}$.

(ii) For any $\vec{t} \in \mathcal{T}$, the set $\{\vec{s} \in \mathcal{T} \mid \vec{s} \sim \vec{t}\}$ is in $\mathbf{A}$.

(iii) For any $t \in \mathcal{T}$, the set $\{s \in \mathcal{T} \mid s \sim t\}$ is in $\mathbf{A}$.

Pr o of. We show that (i) $\Rightarrow$ (iii) $\Rightarrow$ (ii).

For (i) $\Rightarrow\left(\right.$ iii), note that $s \sim t \Leftrightarrow \varphi_{\alpha}^{s}=\varphi_{\alpha}^{t}$, where $\alpha=\operatorname{sr}(\mathcal{T})<\omega_{1}^{\mathcal{T}}$.

(iii) $\Rightarrow$ (ii) follows from the preceding lemma.

For (ii) $\Rightarrow\left(\right.$ i), let $\alpha=\operatorname{sr}(\mathcal{T})$ and let $P_{\vec{t}}=\{\vec{s} \in \mathcal{T} \mid \vec{s} \sim \vec{t}\} \in \mathbf{A}$. We then see that the structure $\left(\mathcal{T}, \vec{t}, P_{\vec{t}}\right) \in \mathbf{A}$. Since

$$
\vec{s} \sim \vec{t} \Leftrightarrow \varphi_{\alpha}^{\vec{s}}=\varphi_{\alpha}^{\vec{t}},
$$

$P_{\vec{t}}$ is definable over the structure $(\mathcal{T}, \vec{t})$ by some formula of $L_{\omega_{1} \omega}$ without any other parameters. By Theorem VII.7.5 of [Ba], $P_{\vec{t}}$ is definable over $(\mathcal{T}, \vec{t})$ by a formula $\psi^{\vec{t}}$ of $L_{\mathbf{A}}$ without any other parameters. Let $\gamma(\vec{t})$ be the rank of $\psi^{\vec{t}}$. Then $\gamma(\vec{t})<\omega_{1}^{\mathcal{T}}$. Moreover, $\vec{s} \sim \vec{t} \Leftrightarrow \varphi_{\gamma(\vec{t})}^{\vec{s}}=\varphi_{\gamma(\vec{t})}^{\vec{t}}$. Let $\gamma_{0}(\vec{t})$ be the least ordinal $\gamma$ such that for any $\vec{s}, \vec{s} \sim \vec{t} \Leftrightarrow \varphi_{\gamma}^{\vec{s}}=\varphi_{\gamma}^{\vec{t}}$. Let $\beta=\sup \left\{\gamma_{0}(\vec{t}) \mid \vec{t} \in \mathcal{T}\right\}$. By boundedness, $\beta<\omega_{1}^{\mathcal{T}}$. But $\operatorname{sr}(\mathcal{T}) \leq \beta$, since if $(\mathcal{T}, \vec{s}) \equiv_{\beta}(\mathcal{T}, \vec{t})$, then $\vec{s} \sim \vec{t}$. This shows that $\operatorname{sr}(\mathcal{T})<\omega_{1}^{\mathcal{T}}$. 
LEMmA 2.3. Let $\mathcal{T}$ be a simple tree with $\operatorname{sr}(\mathcal{T})=\omega_{1}^{\mathcal{T}}$. Then there is a $t \in \mathcal{T}$ with $\operatorname{sr}\left(\mathcal{T}_{t}\right)=\omega_{1}^{\mathcal{T}}$ and infinitely many $s \in \mathcal{T}$ with $s \sim t$.

Proof. Assume not. We then show that $\operatorname{sr}(\mathcal{T})<\omega_{1}^{\mathcal{T}}$. By the preceding lemma it is enough to check that for any $t \in \mathcal{T}$, the set $P_{t}=\{s \in \mathcal{T} \mid s \sim t\}$ is in the least admissible set $\mathbf{A}$ containing $\mathcal{T}$. We show this by induction on $l(t)$. If $l(t)=0$ then $t$ is the root, therefore $P_{t}=\{t\} \in \mathbf{A}$. Suppose $l(t)>0$. If $P_{t}$ is finite, then $P_{t} \in \mathbf{A}$. If $P_{t}$ is infinite, by our assumption $\operatorname{sr}\left(\mathcal{T}_{t}\right)<\omega_{1}^{\mathcal{T}}$. Let $\gamma<\omega_{1}^{\mathcal{T}}$ be a limit ordinal bigger than $\operatorname{sr}\left(\mathcal{T}_{t}\right)$. We claim that $s \sim t \Leftrightarrow p(s) \sim p(t)$ and $\varphi_{\gamma}^{s}=\varphi_{\gamma}^{t}$. To see this, note that the second condition on the right hand side implies that $\mathcal{T}_{s}$ and $\mathcal{T}_{t}$ are isomorphic to each other. Let $\varrho$ be an isomorphism between $\mathcal{T}_{s}$ and $\mathcal{T}_{t}$. Let $\tau$ be a witness for $p(s) \sim p(t)$. If $\tau(s)=t$, there is nothing further to prove. Otherwise, let $s^{*}=\tau^{-1}(t)$ and $t^{*}=\tau(s)$. Then $\varrho^{*}=\tau \varrho^{-1} \tau \mid \mathcal{T}_{s^{*}}$ is an isomorphism between $\mathcal{T}_{s^{*}}$ and $\mathcal{T}_{t^{*}}$. Let

$$
\tau^{\prime}=\varrho \cup \varrho^{*} \cup \tau \mid\left(\mathcal{T} \backslash\left(\mathcal{T}_{s} \cup \mathcal{T}_{s^{*}}\right)\right)
$$

Then $\tau^{\prime}$ witnesses $s \sim t$. This proves the claim. The claim together with the inductive hypothesis shows that $P_{t} \in \mathbf{A}$.

3. The dichotomy for simple trees. In this section we prove the key lemma and derive the strong dichotomy for simple trees. The plan is as follows. We start with an $L_{\omega_{1} \omega}$ sentence $\sigma$ all of whose models are simple trees. Suppose the quantifier rank of this sentence is $\lambda<\omega_{1}$. Suppose $\cong_{\sigma}$ is non-Borel. First we obtain a tree $\mathcal{T}$ in $\operatorname{Mod}(\sigma)$ which is complicated in the sense that it has a large number of automorphisms moving its high rank subtrees around. Then these high rank subtrees of $\mathcal{T}$ can be manipulated so as to obtain a lot of different, in fact pairwise nonisomorphic trees. Finally, the conclusion is that the class of trees $\lambda$-equivalent to $\mathcal{T}$, denoted by $\operatorname{Mod}\left(\equiv_{\lambda} \mathcal{T}\right)$, can code all simple trees in a faithful manner. This coding will provide a Borel reduction of $\mathbb{T}_{\omega}$ into $\operatorname{Mod}\left(\equiv_{\lambda} \mathcal{T}\right)$, thus proving that the class $\operatorname{Mod}(\sigma)$ is Borel complete.

The following lemma is the key lemma which guarantees the existence of a complicated tree described in the preceding paragraph. In the statement of the lemma a subtree of a simple tree $\mathcal{T}$ means a substructure of $\mathcal{T}$ that is closed under $<^{\mathcal{T}}$.

LEMmA 3.1. Let $\mathcal{T}$ be a simple tree with $\operatorname{sr}(\mathcal{T})=\omega_{1}^{\mathcal{T}}$. Then $\mathcal{T}$ contains a subtree $\mathcal{T}^{0}$ with the following properties:

(i) For any $t \in \mathcal{T}^{0}, \operatorname{sr}\left(\mathcal{T}_{t}\right)=\omega_{1}^{\mathcal{T}}$.

(ii) Any $t \in \mathcal{T}^{0}$ has either one child or infinitely many children in $\mathcal{T}^{0}$.

(iii) For any $t \in \mathcal{T}^{0}$, there is $u \in \mathcal{T}^{0}$ such that $u \geq t$ and $u$ has infinitely many children in $\mathcal{T}^{0}$.

(iv) For any $s, t \in \mathcal{T}^{0}$, if $l(s)=l(t)$, then $s \sim_{\mathcal{T}} t$. 
Proof. We construct $\mathcal{T}^{0}$ in $\omega$ many stages. At the end of each stage $n$ we obtain a subtree $\mathcal{T}_{n}^{0}$ of finite height satisfying (i) and (iv). By extending $\mathcal{T}_{n}^{0}$ to $\mathcal{T}_{n+1}^{0}$ we will meet the requirements (ii) and (iii). Let $T_{0}^{0}$ be the empty subtree of $\mathcal{T}$.

By Lemma 2.3, there is a $t_{1} \in \mathcal{T}$ with $\operatorname{sr}\left(\mathcal{T}_{t_{1}}\right)=\omega_{1}^{\mathcal{T}}$ and there are infinitely many $s \in \mathcal{T}$ with $s \sim t_{1}$. Without loss of generality we may assume that there are infinitely many such $s$ with $p(s)=p\left(t_{1}\right)$. This is because, if the assumption fails for $t_{1}$ we can instead consider $p\left(t_{1}\right)$, which also has the property that $\operatorname{sr}\left(\mathcal{T}_{p\left(t_{1}\right)}\right)=\omega_{1}^{\mathcal{T}}$ and there are infinitely many $s$ with $s \sim p\left(t_{1}\right)$. Note that $l\left(p\left(t_{1}\right)\right)=l\left(t_{1}\right)-1$. By an easy induction the procedure stops before we reach the root of $\mathcal{T}$.

Now let $\mathcal{T}_{1}^{0}=\left\{s \in \mathcal{T} \mid s<t_{1}\right.$ or $\left(s \sim t_{1}\right.$ and $\left.\left.p(s)=p\left(t_{1}\right)\right)\right\}$. Then (i) and (iv) hold. For any $t \in \mathcal{T}_{1}^{0}$ that is not a terminal node (ii) and (iii) also follow from the definition. Note that $\mathcal{T}_{t_{1}}$ is definable from $\left(\mathcal{T}, t_{1}\right)$ by a quantifier free formula, so $\omega_{1}^{\mathcal{T}_{t_{1}}} \leq \omega_{1}^{\mathcal{T}}=\operatorname{sr}\left(\mathcal{T}_{t_{1}}\right)$. Hence by Nadel, $\omega_{1}^{\mathcal{T}_{t_{1}}}=\omega_{1}^{\mathcal{T}}$.

Now suppose $\mathcal{T}_{n}^{0}$ has been defined. Fix an arbitrary terminal node $t_{n}$ in $\mathcal{T}_{n}^{0}$. Consider $\mathcal{T}_{t_{n}}$. By Lemma 2.3 and the argument above, we can find $t_{n+1}>t_{n}$ with $\operatorname{sr}\left(\mathcal{T}_{t_{n+1}}\right)=\omega_{1}^{\mathcal{T}}$ such that there are infinitely many $s \in \mathcal{T}$ with $s \sim t$ and $p(s)=p\left(t_{n+1}\right)$. Let $\left\{t_{n}^{i}\right\}_{i \in \omega}$ be an enumeration of all terminal nodes of $\mathcal{T}_{n}^{0}$, with $t_{n}^{0}=t_{n}$. For each $i \in \omega$, let $\tau_{i}$ be a witness for $t_{n} \sim t_{n}^{i}$. Let $S=\left\{s \in \mathcal{T} \mid s<t_{n+1}\right.$ or $\left(s \sim t_{n+1}\right.$ and $\left.\left.p(s)=p\left(t_{n+1}\right)\right)\right\}$. Let $\mathcal{T}_{n+1}^{0}=$ $\mathcal{T}_{n}^{0} \cup \bigcup\left\{\tau_{i}(S) \mid i \in \omega\right\}$. Then (i) and (iv) hold for elements in $\mathcal{T}_{n+1}^{0}$ and (ii) and (iii) hold for elements in $\mathcal{T}_{n}^{0}$.

Eventually let $\mathcal{T}^{0}$ be the increasing union of all $\mathcal{T}_{n}^{0}$. Then $\mathcal{T}^{0}$ is as required.

If $t \in \mathcal{T}^{0}$ has infinitely many children in $\mathcal{T}^{0}$, we say that $t$ is (infinitely) splitting. Otherwise, $t$ has only one child in $\mathcal{T}^{0}$, and we say it is non-splitting. For any $t \in \mathcal{T}^{0}$, let $l^{0}(t)$ denote the cardinality of the set $\left\{s \in \mathcal{T}^{0} \mid s<t\right.$ and $s$ is splitting $\}$ and call it the relative level of $t$ in $\mathcal{T}^{0}$.

Let $\sigma$ be an $L_{\omega_{1} \omega}$ sentence describing an invariant Borel class of simple trees. Let $\lambda>\omega$ be a countable limit ordinal bigger than the quantifier rank of $\sigma$. Suppose that $\cong_{\sigma}$ is non-Borel. By a theorem of Sacks (see [St]) there is a model $\mathcal{T}$ of $\sigma$ with $\operatorname{sr}(\mathcal{T})=\omega_{1}^{\mathcal{T}}>\lambda$. Let $\mathcal{T}^{0}$ be the subtree of $\mathcal{T}$ given by the preceding lemma.

We make use of the following lemma of Steel.

LEMmA 3.2. Let $\mathcal{T}$ be a simple tree with $\operatorname{sr}(\mathcal{T})=\omega_{1}^{\mathcal{T}}$ and let $\lambda<\omega_{1}^{\mathcal{T}}$ be a limit ordinal. Then $\operatorname{id}\left(2^{\omega}\right) \leq_{B} \cong \uparrow \operatorname{Mod}\left(\equiv_{\lambda} \mathcal{T}\right)$.

In the statement, $\operatorname{Mod}\left(\equiv_{\lambda} \mathcal{T}\right)$ is an abbreviation $\operatorname{of} \operatorname{Mod}\left(\varphi_{\lambda}^{\emptyset, \mathcal{T}}\right)$. For a proof of the lemma, see [St]. We define an infinite sequence $\mathcal{U}_{0}, \mathcal{U}_{1}, \ldots, \mathcal{U}_{i}, \ldots$ of simple trees by induction on $i$. For each $i \in \omega$, we fix an arbitrary $t_{i} \in \mathcal{T}^{0}$ 
with $l^{0}\left(t_{i}\right)=i+1$ and $p\left(t_{i}\right)$ splitting. $\mathcal{U}_{i}$ shall be chosen from $\operatorname{Mod}\left(\equiv_{\lambda} \mathcal{T}_{t_{i}}\right)$ so that it is not isomorphic to any $\mathcal{T}_{t}$ for $t \in \mathcal{T}$. In addition, $\mathcal{U}_{i}$ shall not be isomorphic to any $\left(\mathcal{U}_{j}\right)_{t}$ for $j<i$ and $t \in \mathcal{U}_{j}$. Since there are at most countably many trees for $\mathcal{U}_{i}$ to avoid, such a $\mathcal{U}_{i}$ exists by the above lemma.

We are now ready to code $\mathbb{T}_{\omega}$ into $\operatorname{Mod}\left(\equiv_{\lambda} \mathcal{T}\right)$. Given an arbitrary simple tree $\mathcal{S}$, we construct a simple tree $\widetilde{\mathcal{S}}$ by replacing some subtrees of $\mathcal{T}$ by some $\mathcal{U}_{i}$. To do this we first associate a $t(s) \in \mathcal{T}^{0}$ with each $s \in \mathcal{S}$ so that the following properties hold:

(1) $p(t(s))$ is splitting,

(2) $l^{0}(p(t(s)))=l(s)$,

(3) if $s^{\prime} \neq s$, then $t\left(s^{\prime}\right)$ and $t(s)$ are incomparable, and

(4) if $s^{\prime}<s$, then $p\left(t\left(s^{\prime}\right)\right)<p(t(s))$.

Specifically, the assignment can be constructed in a top down manner for elements of $\mathcal{S}$. For each $s \in \mathcal{S}$, we first find a corresponding splitting element in $\mathcal{T}^{0}$ whose relative level is the same as $l(s)$. This element should be greater than $p(t(p(s)))$ and incomparable with any other $t\left(s^{\prime}\right)$. Then we define $t(s)$ to be a child of this element. $\widetilde{\mathcal{S}}$ is then obtained from $\mathcal{T}$ by replacing all $\mathcal{T}_{t(s)}$ by $\mathcal{U}_{l(s)}$. This finishes the construction.

It is easy to see that $\mathcal{S} \mapsto \widetilde{\mathcal{S}}$ is a Borel function. To see that it is a reduction, let $\mathcal{S}_{1}, \mathcal{S}_{2} \in \mathbb{T}_{\omega}$. If $\mathcal{S}_{1} \cong \mathcal{S}_{2}$, then $\widetilde{\mathcal{S}}_{1} \cong \widetilde{\mathcal{S}}_{2}$, since any isomorphism between $\mathcal{S}_{1}$ and $\mathcal{S}_{2}$ naturally induces an isomorphism between $\widetilde{\mathcal{S}}_{1}$ and $\widetilde{\mathcal{S}}_{2}$, which was made possible by Lemma 3.1 (iv). To see that if $\widetilde{\mathcal{S}}_{1} \cong \widetilde{\mathcal{S}}_{2}$ then $\mathcal{S}_{1} \cong \mathcal{S}_{2}$, it is enough to show that $\mathcal{S}$ can in fact be recovered from $\widetilde{\mathcal{S}}$, as done by the following procedure.

First we search for $t \in \widetilde{\mathcal{S}}$ of smallest level with $\widetilde{\mathcal{S}}_{t} \cong \mathcal{U}_{0}$. If no such $t$ exists then $\mathcal{S}$ is empty. If such a $t$ exists then it follows from the definition of the sequence $\left\{\mathcal{U}_{i}\right\}_{i \in \omega}$ that it is unique. This $t$ corresponds to the root of $\mathcal{S}$. Next we remove $\widetilde{\mathcal{S}}_{t}$ from $\widetilde{\mathcal{S}}$ and in the intersection of the remaining part with $\widetilde{\mathcal{S}}_{p(t)}$ search for occurrences of $\mathcal{U}_{1}$ in a similar manner to that above. The occurrences of $\mathcal{U}_{1}$ correspond to the first level elements of $\mathcal{S}$, again by the definition of $\left\{\mathcal{U}_{i}\right\}_{i \in \omega}$. In general, elements of level $n$ in $\mathcal{S}$ are recovered in the $n$th step of this procedure. And eventually all of $\mathcal{S}$ can be recovered. Therefore, if $\widetilde{\mathcal{S}}_{1} \cong \widetilde{\mathcal{S}}_{2}$, then the above procedures recover isomorphic trees, thus $\mathcal{S}_{1} \cong \mathcal{S}_{2}$.

In effect we have proven the following theorem.

THEOREM 3.1. Let $\sigma$ be an $L_{\omega_{1} \omega}$ sentence all of whose models are simple trees. Then either $\cong_{\sigma}$ is Borel or $\operatorname{Mod}(\sigma)$ is Borel complete.

4. The main theorem. In this section we prove the main dichotomy theorem for mono-unary algebras. 
THEOREM 4.1. Let $\sigma$ be an $L_{\omega_{1} \omega}$ sentence such that $\operatorname{Mod}(\sigma) \subseteq \mathbb{U}$. Then either $\cong_{\sigma}$ is Borel or else $\operatorname{Mod}(\sigma)$ is Borel complete.

Recall that our official language $L$ consists of a binary relation symbol $R$ which can be viewed as a partial function. Let us denote this partial function by $F$, i.e., $F(x)=y \Leftrightarrow R(x, y)$. We will use the notation $F^{n}, n \in \omega$, in the intuitive sense:

$F^{0}(x)=x$,

$F^{n}(x)=y \Leftrightarrow \exists x_{1} \ldots \exists x_{n}\left(R\left(x, x_{1}\right) \wedge \ldots \wedge R\left(x_{n-1}, x_{n}\right) \wedge x_{n}=y\right)$, for $n>0$.

Then one can define a partial order $<_{F}$ as follows:

$$
x<_{F} y \Leftrightarrow \exists n>0\left(F^{n}(y)=x\right) \wedge \forall m\left(F^{m}(x) \neq y\right) .
$$

Let $M \in \mathbb{U}$. For any $x, y \in M, x$ and $y$ are said to be connected if there are $n, m \in \omega$ such that $F^{n}(x)=F^{m}(y)$. For each $x \in M$, the (connected) component of $x$ is $C_{x}={ }_{\text {def }}\{y \in M \mid x$ and $y$ are connected $\}$. It is easy to check that being connected is an equivalence relation and the components are the equivalence classes, therefore giving a partition of $M . M$ is connected if every pair of elements in $M$ are connected. There are only three kinds of components $C$, as follows:

Type I: There is no $<_{F}$-minimal element in $C$. With respect to $<_{F}, C$ is an infinite tree without root. This happens when $F(x)$ is always defined for any $x \in C$, and $F^{n}(x) \neq x$ for any $n>0$ and $x \in C$.

Type II: There are more than one $<_{F}$-minimal elements in $C$. In this case the $<_{F}$-minimal elements constitute a finite directed cycle with respect to $F$. This happens when $F(x)$ is always defined for any $x \in C$ and $F^{n}(x)=x$ for some $x \in C$ and $n>1$.

Type III: There is a $<_{F}$-least element in $C$. With respect to $<_{F}, C$ is just a simple tree. This happens either when $F(x)=x$ for some $x \in C$ or when $F(x)$ is not defined for some $x \in C$. In either case the special element is the root.

For each $x \in M$, let $T_{x}$ be the structure with domain

$$
\left\{y \in M \mid \exists n\left(F^{n}(y)=x \wedge \forall m<n \forall k>0\left(F^{m+k}(y) \neq F^{m}(y)\right)\right\}\right.
$$

and the partial order $<_{F}$. Then $T_{x}$ is always a simple tree.

Lemma 4.1. Let $M \in \mathbb{U}$ be such that $\operatorname{sr}(M)=\omega_{1}^{M}$. Then there is $x \in M$ such that $\operatorname{sr}\left(T_{x}\right)=\omega_{1}^{M}$.

Proof. We first claim that there must be a component $C$ with $\operatorname{sr}(C)=$ $\omega_{1}^{M}$. Assume not. Then for any $x \in M, \operatorname{sr}\left(C_{x}\right)<\omega_{1}^{M}$. By boundedness, there is a limit ordinal $\gamma<\omega_{1}^{M}$ such that $\operatorname{sr}\left(C_{x}\right)<\gamma$ for all $x \in M$. We demonstrate that $\operatorname{sr}(M)<\gamma+\omega$, hence a contradiction. For this it suffices to show that for any $\vec{a}, \vec{b} \in M$, if $(M, \vec{a}) \equiv_{\gamma+\omega}(M, \vec{b})$ then there is an 
automorphism $\tau$ of $M$ with $\tau(\vec{a})=\vec{b}$. Without loss of generality we may assume all elements in $\vec{a}$ are in a single component, say $C_{0}$. Then it follows that all elements in $\vec{b}$ are in a single component as well, say $C_{1}$. Moreover $\left(C_{0}, \vec{a}\right) \equiv_{\gamma}\left(C_{1}, \vec{b}\right)$. Since $\operatorname{sr}\left(C_{0}\right)<\gamma$, there is an isomorphism $\tau: C_{0} \rightarrow C_{1}$ with $\tau(\vec{a})=\vec{b}$. It is then obvious that $\tau$ can be extended to an automorphism of $M$.

Therefore we may assume that $M$ is connected. To see that there is $x \in M$ with $\operatorname{sr}\left(T_{x}\right)=\omega_{1}^{M}$, we consider the three types of $M$. If $M$ is of type III, there is nothing to prove. If $M$ is of type II, then $M$ is the union of finitely many simple trees with their roots tied together by a cycle. One of these simple trees is as required. The only nontrivial case is when $M$ is of type I.

Suppose $M$ is of type I and suppose that for any $x \in M, \operatorname{sr}\left(T_{x}\right)<\omega_{1}^{M}$. Then by boundedness there is a limit ordinal $\gamma<\omega_{1}^{M}$ such that $\operatorname{sr}\left(T_{x}\right)<\gamma$, $\forall x \in M$. We may assume that $\omega \gamma=\gamma$. This ensures that $\operatorname{sr}\left(T_{x},<_{F}\right)<\gamma$ is equivalent to $\operatorname{sr}\left(T_{x}, R\right)<\gamma$, so we can omit the relation in the following computations. We shall demonstrate that $\operatorname{sr}(M)<\gamma+\omega$, hence a contradiction. For this it suffices to show that for any $\vec{a}, \vec{b} \in M$, if $(M, \vec{a}) \equiv_{\gamma+\omega}(M, \vec{b})$ then there is an automorphism $\tau$ of $M$ with $\tau(\vec{a})=\vec{b}$.

Suppose $\vec{a}$ and $\vec{b}$ are $n$-tuples. Let $x_{0} \in M$ be maximal so that $x_{0} \leq_{F} a_{i}$ for all $i<n$. Let $y_{0} \in M$ be the corresponding element for $\vec{b}$. Then it follows that $\left(T_{x_{0}}, \vec{a}\right) \equiv_{\gamma}\left(T_{y_{0}}, \vec{b}\right)$. Since $\operatorname{sr}\left(T_{x_{0}}\right), \operatorname{sr}\left(T_{y_{0}}\right)<\gamma$, there is an isomorphism $\varrho_{0}$ between $T_{x_{0}}$ and $T_{y_{0}}$ so that $\varrho_{0}(\vec{a})=\vec{b}$. Now for $n>0$, let $x_{n}=F^{n}\left(x_{0}\right)$ and $y_{n}=F^{n}\left(y_{0}\right)$. It follows from the assumption $(M, \vec{a}) \equiv_{\gamma+\omega}$ $(M, \vec{b})$ that for each $n>0, T_{x_{n}} \equiv_{\gamma} T_{y_{n}}$. Hence for each $n>0$ there is an isomorphism $\varrho_{n}$ between $T_{x_{n}}$ and $T_{y_{n}}$. By the proof of Lemma 2.3 we may assume that $\varrho_{n} \mid T_{x_{n-1}}=\varrho_{n-1}$ for all $n>0$. Finally notice that $M=$ $\bigcup_{n \in \omega} T_{x_{n}}=\bigcup_{n \in \omega} T_{y_{n}}$. Let $\tau=\bigcup_{n \in \omega} \varrho_{n}$. Then $\tau$ is an automorphism of $M$ with $\tau(\vec{a})=\vec{b}$.

Now the proof of Theorem 4.1 follows the same line of proof as Theorem 3.1.

Proof of Theorem 4.1. Let $\sigma$ be an $L_{\omega_{1} \omega}$ sentence describing an invariant Borel subclass of $\mathbb{U}$. Let $\lambda>\omega$ be a countable limit ordinal bigger than the quantifier rank of $\sigma$. Suppose that $\cong_{\sigma}$ is non-Borel. By Sacks' Theorem there is a model $M$ of $\sigma$ with $\operatorname{sr}(M)=\omega_{1}^{M}>\lambda$. By Lemma 4.1 there is $x \in M$ such that $\operatorname{sr}\left(T_{x}\right)=\omega_{1}^{M}=\omega_{1}^{T_{x}}>\lambda$. Therefore by the proof of Theorem 3.1 there is a Borel reduction of $\mathbb{T}_{\omega}$ into $\operatorname{Mod}\left(\equiv_{\lambda} T_{x}\right)$. This induces an embedding of $\mathbb{T}_{\omega}$ into $\operatorname{Mod}\left(\equiv_{\lambda} M\right)$, provided that the previous embedding was chosen so that the isomorphic types of all $T_{y}, y \in M$, are avoided by the coding blocks. This shows that $\operatorname{Mod}\left(\equiv_{\lambda} M\right)$, hence $\cong_{\sigma}$, is Borel complete. 
5. Corollaries and remarks. Theorem 4.1 and the Glimm-Effros dichotomy for Borel equivalence relations ([HKL]) immediately yield the following corollary.

COROLlary 5.1 (The Glimm-Effros dichotomy). Let $\sigma$ be an $L_{\omega_{1} \omega}$ sentence such that $\operatorname{Mod}(\sigma) \subseteq \mathbb{U}$. Then either $\cong_{\sigma} \leq_{B} \mathrm{id}(\mathbb{R})$ or else $E_{0} \leq_{B} \cong_{\sigma}$.

The Glimm-Effros dichotomy in turn implies the well known result about the number of countable models for an invariant Borel class of mono-unary algebras.

Corollary 5.2 (Vaught Conjecture, Marcus-Miller-Steel). Let $\sigma$ be an $L_{\omega_{1} \omega}$ sentence such that $\operatorname{Mod}(\sigma) \subseteq \mathbb{U}$. Then $\sigma$ has either countably many or perfectly many models up to isomorphism.

Moreover, we obtain a stronger result about first-order theories of monounary algebras from the proofs of preceding sections.

THEOREM 5.3. Let $T$ be a first-order theory of mono-unary algebras. Then the following statements are equivalent:

(i) There is a model $M$ of $T$ with $\operatorname{sr}(M)=\omega_{1}^{M}$.

(ii) For any ordinal $\alpha<\omega_{1}$, there is a model $M$ of $T$ with $\operatorname{sr}(M)=$ $\omega_{1}^{M}>\alpha$.

(iii) $\cong_{T}$ is non-Borel.

(iv) $\operatorname{Mod}(T)$ is Borel complete.

Proof. (i) $\Rightarrow$ (iv) by the proof of Theorem 4.1. (iv) $\Rightarrow$ (iii) by the result of Friedman and Stanley that the isomorphism of any Borel complete class is indeed $\boldsymbol{\Sigma}_{1}^{1}$-complete. (iii) $\Rightarrow$ (ii) by Sacks' Theorem. (ii) $\Rightarrow$ (i) is obvious.

We now turn to the question of Friedman and Stanley. For each countable ordinal $\alpha<\omega_{1}$, let $\mathbb{S}_{\alpha}$ be the class of well founded trees of rank $\alpha$. Then $\left\{\mathbb{S}_{\alpha}\right\}_{\alpha \in \omega_{1}}$ is an $\omega_{1}$-sequence of invariant Borel classes of simple trees. It is known that

$$
\mathbb{S}_{0}<_{B} \mathbb{S}_{1}<_{B} \mathbb{S}_{2}<_{B} \ldots<_{B} \mathbb{S}_{\alpha}<_{B} \ldots
$$

Hence the isomorphism relations are strictly increasing in terms of Borel reducibility. Another way to view the tower is that $\mathbb{S}_{2}$ can be identified with $\omega, \mathbb{S}_{3}$ with the reals (in the Cantor space), $\mathbb{S}_{4}$ with the space of countable sets of reals, $\mathbb{S}_{5}$ with the space of countable sets of countable sets of reals, etc. The isomorphism relations between trees are then viewed as the identity relations of the corresponding objects. Moreover, each of the spaces can be given a topology which is Polish.

The Scott sentence of any countable model can be viewed as an element of $\mathbb{S}_{\alpha}$, where $\alpha$ is the Scott rank of the model. Thus the Scott analysis assigns to each countable model an element of some classes in the above tower. When we consider an invariant Borel class whose isomorphism relation is Borel, 
this assignment is then a Borel reduction by boundedness. This shows that the Friedman-Stanley tower dominates all Borel isomorphism relations on invariant Borel classes of countable models.

An invariant Borel class $\mathbb{A}$ is $\alpha$-complete if $\mathbb{S}_{\alpha} \leq_{B} \mathbb{A}$. The question of Friedman and Stanley is whether an invariant Borel class is Borel complete if it is $\alpha$-complete for all $\alpha<\omega_{1}$. Our theorem answers the question for invariant Borel classes of mono-unary algebras.

Corollary 5.4. Let $\mathbb{A}$ be an invariant Borel class of mono-unary algebras. If $\mathbb{A}$ is $\alpha$-complete for all $\alpha<\omega_{1}$, then $\mathbb{A}$ is Borel complete.

Proof. If $\cong \mathbb{A}$ is Borel then $\mathbb{A} \leq_{B} \mathbb{S}_{\alpha}$ for some $\alpha<\omega_{1}$, by the above observation about Scott analysis. The hypothesis then implies that $\cong \backslash \mathbb{A}$ is non-Borel. Therefore $\mathbb{A}$ is Borel complete by Theorem 4.1.

Note that Theorem 4.1 cannot be generalized to arbitrary countable models. In fact, let $\mathbb{A}$ be the class of countable abelian torsion groups. Then $\cong \mathbb{A}$ is not Borel (Theorem 6 of [FS]), yet $\mathbb{S}_{4} \not_{B} \mathbb{A}$ (Theorem 5 of [FS]). This latter fact was strengthened in $[\mathrm{HK}]$ to $E_{0} \mathbb{Z}_{B} \cong \uparrow \mathbb{A}$. Hence $\mathbb{A}$ is not Borel complete, in fact the Glimm-Effros dichotomy fails for it.

From a more abstract point of view, Theorem 4.1 can be viewed as a first step toward a complete classification for $\cong_{\sigma}$ with respect to Borel reducibility. A further result along this line of research is contained in [Ga], which states that for any invariant Borel class $\mathbb{B}$ with $\cong \uparrow \mathbb{B}$ Borel, there is an invariant Borel class $\mathbb{A}$ of mono-unary algebras such that $\mathbb{A} \leq_{B} \mathbb{B} \leq_{B} \mathbb{A}$. However, it is not clear what $\cong_{T}$ can achieve for first-order theories $T$ of mono-unary algebras.

\section{References}

[Ba] J. Barwise, Admissible Sets and Structures: an Approach to Definability Theory, Perspectives in Math. Logic, Springer, Berlin, 1975.

[BK] H. Becker and A. S. Kechris, The Descriptive Set Theory of Polish Group Actions, London Math. Soc. Lecture Note Ser. 232, Cambridge Univ. Press, Cambridge, 1996.

[FS] H. Friedman and L. Stanley, A Borel reducibility theory for classes of countable structures, J. Symbolic Logic 54 (1989), 894-914.

[Ga] S. Gao, The isomorphism relation between countable models and definable equivalence relations, Ph.D. dissertation, UCLA, 1998.

[HKL] L. Harrington, A. S. Kechris and A. Louveau, A Glimm-Effros dichotomy for Borel equivalence relations, J. Amer. Math. Soc. 3 (1990), 903-928.

[HK] G. Hjorth and A. S. Kechris, Analytic equivalence relations and Ulm-type classifications, J. Symbolic Logic 60 (1995), 1273-1300.

[Ma] L. Marcus, The number of countable models of a theory of one unary function, Fund. Math. 58 (1980), 171-181. 
[Sa] R. Sami, Polish group actions and the Vaught Conjecture, Trans. Amer. Math. Soc. 341 (1994), 335-353.

[St] J. R. Steel, On Vaught's Conjecture, in: Cabal Seminar 76-77, Lecture Notes in Math. 689, Springer, Berlin, 1978, 193-208.

Department of Mathematics

California Institute of Technology

Pasadena, CA 91125, U.S.A.

E-mail: sugao@its.caltech.edu

Received 2 March 1999;

in revised form 7 September 1999 